Methods Data were obtained from the United States Cancer Statistics (USCS) Public Use Databases from 2001 to 2017. SEER*Stat 8.3.9, Joinpoint regression program 4.8.0.1, and Excel were used to calculate the incidence and trends. The age-adjusted incidence was calculated by WHO 2000 standard population.

Results In 2017, the incidence of ovarian and uterine carcinosarcoma was 0.25 and 1.03 per 100,000 women, respectively. of all carcinosarcomas, the incidence was highest in Blacks at 2.7 followed by 1.1 and 0.82 in Whites and Hispanics, respectively. Over the last 17 years, the annual incidence of carcinosarcoma has increased across all races, however for Black and Hispanic women that rate is increasing 2.5 times compared to Whites. More specifically, the percent increase was $3.7 \%$ per year for Blacks, $4 \%$ for Hispanics, and $1.5 \%$ for Whites. Black women in the $70-74$ age group had the highest incidence of uterine carcinosarcoma at 21.6/100,000; while 60-64 year old Black women had the greatest percent increase of uterine carcinosarcoma at $3.3 \%$ per year.

Conclusions The incidence of ovarian and uterine carcinosarcoma is increasing across all races, but most notably for older Black women.

\section{EPV246/\#242 THE GENDER IMBALANCE IN GYNECOLOGIC ONCOLOGY AUTHORSHIP AND EDITORIAL BOARDS}

${ }^{1} \mathrm{SJ}$ Mah, ${ }^{2} \mathrm{M}$ Makkar*, ${ }^{2} \mathrm{~T}$ Anpalagan, ${ }^{2} \mathrm{~K}$ Huang, ${ }^{1} \mathrm{C}$ Reade, ${ }^{3} \mathrm{M}$ Cachia, ${ }^{1} \mathrm{JMV}$ Nguyen. ${ }^{1}$ McMaster University, Juravinski Hospital and Cancer Centre, Gynecologic Oncology, Hamilton, Canada; ${ }^{2}$ McMaster University, Michael G. Degroote School of Medicine, Hamilton, Canada; ${ }^{3}$ McMaster University, Department of Health Research Methods, Evidence, and Impact, Hamilton, Canada

\subsection{6/ijgC-2021-IGCS.317}

Objectives Despite increased participation of women in Medicine in recent decades, there remains gender disparity in Academic Medicine. Our objective was to examine gender diversity in authorship and editorial boards of 2 prominent peer-reviewed Gynecologic Oncology journals.

Methods We performed a bibliometric analysis of original articles published in Gynecologic Oncology(GO) and International Journal of Gynecologic Cancer(IJGC), comparing 20162020 to two decades prior (1996). First names and photographs from institutional websites were used to identify subjective gender. Gender distribution was compared using chisquare tests.

Results We included 3022 original articles between 20062020, and 201 in 1996. Gender was identified for $93 \%$ of
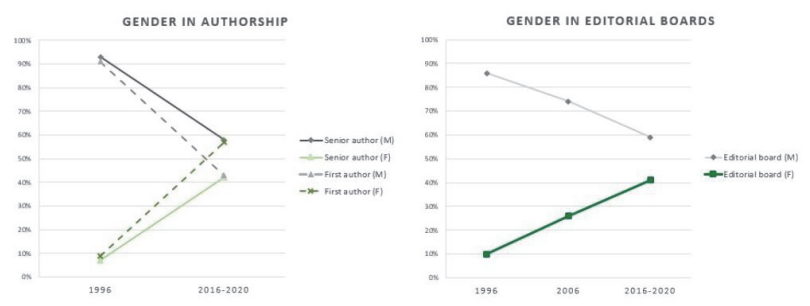

Abstract EPV246/\#242 Figure 1 first and $94.5 \%$ of senior(last) authors. Trends of gender representation in authorship and editorial boards over time are presented in the graphs below. Men were overrepresented as senior authors across both study periods: $93 \%$ in 1996, and $58 \%$ in 2016-2020. Over time, representation of women as senior authors increased $(7 \%$ in 1996, 42\% in 2016-2020, $\mathrm{p}<0.00001)$. This trend was also observed in first authorship (9\% in 1996, 57\% in 2016-2020, p<0.00001). Men continued to comprise the majority of editorial board members (86\% in $1996,59 \%$ in 2020).

Conclusions There is an encouraging trend in female authorship, likely reflective of increased representation in the workforce. Nevertheless, gender disparity persists. This underlines an opportunity for the academic publishing community to participate in advocacy and decisive action to close the 'gender gap'. The impact of the COVID-19 pandemic on this publication gap is being assessed by our group.

\section{EPV247/\#348 RACIAL DISPARITIES IN OVARIAN CANCER SURVIVAL IN THE UNITED STATES DESPITE ADHERENCE TO NATIONAL GUIDELINE CARE}

1) Sussell, ${ }^{1} \mathrm{C}$ Ng, ${ }^{1}$ I Abbass, ${ }^{2} \mathrm{~T}$ Tunnage ${ }^{*},{ }^{3} \mathrm{~L}$ Cassoli, ${ }^{4} \mathrm{C}$ Chan. ${ }^{1}$ Genentech, Inc., Evidence For Access, Us Medical Affairs, South San Francisco, USA; ${ }^{2}$ New York University, Gynecologic Oncology, New York, USA; ${ }^{3}$ Genentech, Inc., Us Medical Affairs, South San Francisco, USA; ${ }^{4}$ California Pacific Medical Center Research Institute, Gynecologic Oncology, San Francisco, USA

\subsection{6/ijgc-2021-IGCS.318}

Objectives To assess the relationship between race, receipt of National Comprehensive Cancer Network guideline adherent care (AC), and overall survival in a population of White and Black ovarian cancer (OC) patients in the United States.

Methods We used data for ovarian cancer patients diagnosed 2011-2019 in Flatiron Health, a longitudinal database spanning $>265$ cancer clinics and $>2 \mathrm{M}$ US patients. We defined $\mathrm{AC}$ as surgery and $\geq 6$ cycles of platinum/taxane doublet chemotherapy, with variation based on NCCN guidelines in effect at diagnosis. We modeled overall survival as a function of $\mathrm{AC}$, race, and clinical and demographic factors using Cox regression.

Results of 2,138 patients (median: 67 years; range 20-84), 1974 (92\%) were White and 164 (8\%) were Black. Only $45.3 \%$ and $45.7 \%$ of White and Black patients received AC. In multivariate analysis, $\mathrm{AC}$ in the overall population (HR $0.557, \mathrm{p}<0.001)$ was associated with improved survival, while age (HR 1.028, p<0.001), stage IV at diagnosis (HR 1.471, $\mathrm{p}<0.001$ ), and living in the Southern/Midwestern US (HR 1.366 and 1.342, respectively, $p=0.002$ and $p=0.013$ ) were associated with worsened survival. AC predicted improved survival for White patients (HR 0.540, p<0.001) but not for Black patients (HR 0.819, $\mathrm{p}=0.369$ ).

Conclusions Adherent care (AC) predicted improved survival for White patients with OC, but not their Black counterparts. It is unclear whether differences result from the small number of Black patients in our sample, or from racial differences in the individual components of AC.The relationship between race, $\mathrm{AC}$, and survival should be further investigated. 